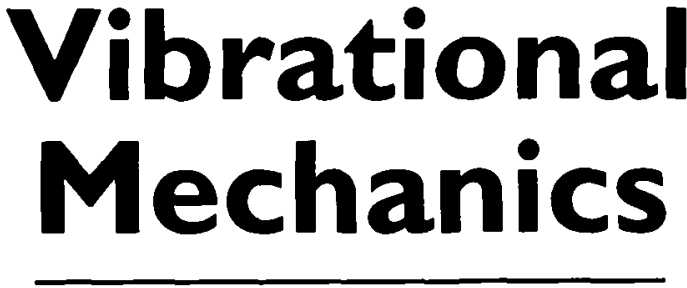

Nonlinear Dynamic Effects, General Approach, Applications 
This page is intentionally left blank 


\title{
Vibrational Mechanics \\ Nonlinear Dynamic Effects, General Approach, Applications
}

\section{Iliya I Blekhman}

\author{
Russian Academy of Sciences \\ St. Petersburg \\ Translated by \\ Minna Perelman
}




\section{Published by}

World Scientific Publishing Co. Pte. Ltd.

P O Box 128, Farrer Road, Singapore 912805

USA office: Suite 1B, 1060 Main Street, River Edge, NJ 07661

UK office: 57 Shelton Street, Covent Garden, London WC2H 9HE

\section{British Library Cataloguing-in-Publication Data}

A catalogue record for this book is available from the British Library.

\section{VIBRATIONAL MECHANICS \\ Nonlinear Dynamic Effects, General Approach, Applications}

Copyright $\odot 2000$ by World Scientific Publishing Co. Pte. Ltd.

All rights reserved. This book, or parts thereof, may not be reproduced in any form or by any means, electronic or mechanical, including photocopying, recording or any information storage and retrieval system now known or to be invented, without written permission from the Publisher.

For photocopying of material in this volume, please pay a copying fee through the Copyright Clearance Center, Inc., 222 Rosewood Drive, Danvers, MA 01923, USA. In this case permission to photocopy is not required from the publisher.

ISBN 981-02-3890-8

Printed in Singapore by Uto-Print 
Dedicated to the memory of Nina 
This page is intentionally left blank 


\section{Preface to the Russian Edition}

This book has two aims. The first aim is to describe a number of wonderful phenomena caused by the action of vibration on nonlinear mechanical systems. Here is a short list of such phenomena: vibrational displacement - the directed on the average "slow" motion or the change in the state of the system affected by "fast" undirected on the average mechanical actions (vibration); the change in the physical and mechanical properties of a body under vibration with regard to slow actions; the transformation of equilibrium states, in particular their stabilization and destabilization under the action of vibration; the change in free oscillation frequencies of the system, caused by vibration; vibrational maintenance of rotation and self-synchronization of unbalanced rotors having an unexpected analogue in the peculiarities of the motion of celestial bodies; the seeming change in both magnitude and direction of the gravitational force. That enumeration is far from being complete. Many of those phenomena are widely used in technology and engineering. Still more effective use of such phenomena is expected in the nearest future. The author hopes this book will contribute to it.

The second, and perhaps the main aim of the book is to propose a general mechanical and mathematical approach to the description and investigation of this class of phenomena - the approach which may be called vibrational mechanics. Vibrational mechanics is a mechanics for the observer interested only in the "slow" motions of the system. Such motions appear together with the fast motions in a non-linear system under vibration and are, as a rule, of the main practical interest. It has been found that this observer, whom we call the observer $\mathbf{V}$ to distinguish him from the "ordinary" observer whom we will call the observer $\boldsymbol{O}$, in order to make a correct description of the behaviour of the system must add to all the slow forces, acting upon the system, certain supplementary slow forces, called vibrational forces. These forces are calculated according to certain rules. Thus, the vibrational mechanics is in some sense analogous to the mechanics of a relative motion.

All the above-mentioned phenomena can be easily and naturally explained by the appearance of vibrational forces. On the other hand, the disregard of those forces leads to misunderstanding and errors, which happened in the past and are still happening now. We will discuss them in this book.

Vibrorheology is an important part of vibrational mechanics. It mainly studies the change caused by vibration in the rheological characteristics of bodies with regard to the slow actions, and it also studies the corresponding slow motions. 
Vibrational mechanics and vibrorheology play an important role in the new section of the applied theory of oscillations which has been formed in recent years - the theory of vibrational processes and vibrational devices. This theory studies the regularities of excitation and effects of vibration in different mechanical systems. It also embraces the theory of the machines in which vibration is used for beneficial purposes.

A few more words about the positions of the observers $\mathbf{O}$ and $\mathbf{V}$. - positions which are widely used in this book and in some illustrations are marked by symbols and The letters $O$ and $V$ are the initial letters of the words "ordinary" and "vibrational". In the world, perceived by the observer $\mathrm{O}$, the ordinary laws of mechanics are valid in their direct form. Making and solving differential equations of motion, based on those laws, that observer gives correct descriptions of the phenomena, considered in the book. But his descriptions are rather intricate, with unnecessary details, as a result of which the interpretation of the results is sometimes quite embarrassing.

The observer $\mathbf{V}$, as was mentioned, deliberately "does not notice" any fast forces or fast motions, though he does not forget that they do exist in reality. Staying within the frames of the "ordinary" mechanics, to make his descriptions correct, he must add to all the slow forces also the vibrational forces. The world of the observer $\mathbf{V}$ is much simpler than that of the observer $\mathbf{O}$. In particular, the multidimensional system may be seen by him as a system of much smaller dimensions, an essentially non-conservative system may seem to him to be conservative, the discontinuous system may be seen by him as continuous or "smooth" and so on. As was mentioned, for the observer V vibrational mechanics and vibrorheology are mechanics and rheology respectively . In connection with the mistakes, which are often made when analyzing such phenomena, we can also speak of the position of the observer $\mathbf{W}$ (the first letter of the word "wrong"). He fails to take into account certain rather important circumstances. This observer either does not notice vibration and the fast forces, acting in the system, or does not take into consideration any possible consequences of their presence. So when considering the slow motions, he does not take into account a possibility of the appearance of vibrational forces. The world of this observer is full of "miracles", riddles and paradoxes. Trying to explain them he sometimes begins to doubt the validity of the basic laws of mechanics - the law of conservation of energy, the law of action and reaction. He believes that under the action of vibration the weight of the body changes, that the velocity of the center of inertia of the system may change only at the expense of internal forces, etc. 
However, the position of the observer $\mathbf{W}$ may sometimes also prove useful. In inventors' activities a temporal disregard of the laws of physics and mechanics, which hinder in attaining their aim, is an effective method of finding new technical solutions. We mean here the so called fantastic analogy, proposed by Gordon [176].

Now a few words about the terms vibrational mechanics and vibrorheology. Their introduction seems to be justified, first, by the peculiarity of the class of phenomena that are to be considered and, secondly, by the availability of a common methodical approach to the investigation of these effects. These terms, used by the author in his presentations and publications met no objections on the part of his colleagues $[89,92,95,100]$. Equations, describing the slow motions, are called by us the main equations of vibrational mechanics. It is surprising and remarkable that for a comparatively wide class of systems these equations can be written in the form, typical of the potential system with dissipation, while the initial system is essentially non-potential. Systems of this type are named by us potential on the average dynamic systems. A number of important practical problems, in particular problems on synchronization (resonances) under the rotation of solid bodies, are reduced to studying those systerns.

Vibrational mechanics is considered in this book as a special case of a more general concept which can be named mechanics of systems with hidden motions. Both in the process of mechanical-mathematical modeling real systems and in striving to simplify the models, one has a natural desire "not to see", "to partly ignore" certain components of motions of the system and even some degrees of freedom which seem to be of minor importance. The question arises: by which mechanics should the observer be guided lest he should come into conflict with reality? The answer is analogous to the previous one: certain additional forces should be introduced into the equations, describing the motions which are to be considered. Just like above, the mechanics of systems with partly ignored motions can be interpreted from the points of view of the three observers $\mathbf{O}^{\prime}$, $\mathbf{V}^{\prime}$ and $\mathbf{W}^{\prime}$ sinilar to observers $\mathbf{O}, \mathbf{V}$ and $\mathbf{W}$. It is remarkable that vibrational mechanics is connected with both the classic works on mechanics and modern investigations. By the former we mean the works by Thomson, Tait, and Routh on mechanics of systems with cyclic coordinates, the works by Reynolds on the theory of turbulence, by Poincare and Lyapunov on celestial mechanics and theory of the stability of motion, by Krylov, Bogolyubov, Mitropolsky and Malkin on methods of averaging and the theory of periodic solutions of differential equations with a srnall parameter. By the latter we mean the works by Kapitsa and his followers on the behavior of a pendulum with a vibrating 
axis of suspension, on the theory of vibrational processes and devices and also on the problem of resonances in the Solar system. That common character of ideas and their continuity has been systematically reflected in this book.

The first part of the book is devoted to the theoretical basis of vibrational mechanics, in particular to the description of the general approach, mentioned above, which is mainly based on the so called method of direct separation of motions. The main statements have been formulated here as theorems. It should be noted however that the elaboration of the mathematical apparatus of vibrational mechanics cannot as yet be considered completed. Other chapters of the book are devoted to numerous applications of vibrational mechanics.

The book is provided with a detailed table of contents and detailed captions to figures. It seems to us both of them will be helpful to the readers.

The book is meant first of all for the specialists in both theoretical and applied mechanics, in the theory of non-linear oscillations and vibrational technology. It may also prove to be useful for mathematicians involved in the theory of ordinary differential equations as a source of some new problems.

While working at the concept and apparat us of vibrational mechanics I had a happy chance to discuss the main points with V.I.Babitsky, V.V.Beletsky, V.V.Koslov, M.Z. Kolovsky, P.S.Landa, L.G.Loytsyansky, Ja.G.Panovko, A.A.Pervosvansky, V.V.Rumyantsev, O.V.Savinov, G.Ju.Stepanov, and K.V.Frolov. My young colleagues E.B.Kremer, O.Z.Malakhova, A.V.Petchenev. A.Ya. Fidline and N.P.Yaroshevich both on my request and on their own initiative made a number of complicated researches the results of which have been reflected in the book. Besides, O.Z.Malakhova read the manuscript very attentively and helped to eliminate some drawbacks. While reviewing the book, important remarks were made by M.Z.Kolovsky. I express my deep gratitude to those scientists.

I appreciate the unfailing understanding and support of my colleagues and the management of the Research and Design Institute of Mechanical Treatment of Mineral Resources ("Mekhanobr", St-Petersburg) which made it possible for me to devote myself to this most interesting work.

I am greatly thankful to my wife Nina Granat who passed away when the work on the book was practically completed. A gifted researcher herself (the reader will find some results of her work in the book), Nina selflessly released me from any domestic care. To Nina's fond memory this book is dedicated.

November 29, 1991, St.Petersburg 


\section{Preface to the English Edition}

I am thankful to the World Scientific Publishing Co. for the opportunity to present my book to the English readers.

Five years have passed since the publication of this book in Russia [125]. The ideas and results stated there have been essentially developed and supplemented during that time. In this English edition of the book the author tended to at least partially reflect those developments:

- item 3.3 has been added, which is devoted to the characteristics of the advantages and limitations of the approach and methods stated in the book;

- item 5.3 has been added, which gives a short review of the results referring to the stability of composite pendulums with a vibrating axis of suspension, including the follower-loaded ones;

- item 7.8 was supplemented by the general definition of synchronization;

item 10.6, devoted to the vibrational pumps and coaches has been considerably enlarged;

a new chapter had been introduced. This chapter (chapter 18) contains some ideas on the problem of controlling the properties of the nonlinear systems by means of vibration. Here the new approach to the creation of the materials with given dynamic properties - the so called dynamic materials - is discussed most thoroughly;

- the bibliography contains additional references to both the latest investigations and to those made before, which unfortunately escaped the attention of the author when the book was being prepared.

In 1996 the author delivered a PhD course of lectures on vibrational mechanics at the Department of Solid Mechanics, headed by Professor Pauli Pederson, at the Technical University of Denmark (DTU).

Then similar lectures were delivered at the Institute of the Problems of Mechanical Engineering of the Russian Academy of Sciences; at the St.Petersburg Technical University (the former Polytechnical Institute) and at the Marine Technical University.

Professors D. A. Indeitsev, M. Z. Kolovsky and S. V. Sorokin, who had invited me, and their disciples as well as their colleagues at the DTU made an attentive and interested audience. The contact with them proved to be 
both pleasant and useful. Some of them, including Professors J. J. Thomsen, S. V. Sorokin and their pupils J. S. Jensen, D. M. Tchernyak and E. C. Miranda found it interesting to make their own investigations of that problem. References to their investigations and short reviews of them are given in the book.

The author's contacts with Professors A. L. Fradkov, K. A. Lurie, and L. Sperling were both very useful and very pleasant.

I am deeply grateful to all those scientists.

I want to thank Associate Professor Minna Perelman for her careful translation in the process of which some fragments of the text were improved.

I am also thankful to my colleague Larissa Titova for her valuable assistance in preparing the manuscript.

I am grateful to Professor Mikhail Levinshtein for his helpful advice.

July 30, 1999, St.Petersburg

I.Blekhman 
Contents

Preface to the Russian Edition . . . . . . . . . . . . . . . . . vii

Preface to the English Edition . . . . . . . . . . . . . xi

I Fundamentals of Theory of Vibrational Mechanics 1

1 Introduction. Subject-Matter of Vibrational Mechanics 3

1.1 Oscillatory Processes in Nature and Engineering . . . . . 3

1.2 Damaging Vibration and Useful Vibration. Vibrational Engineering ................ . . 3

1.3 The Theory of Vibrational Processes and Devices - a New Section of the Applied Theory of Oscillations . . . . . . . 5

1.4 On the Effects Caused by the Action of Vibration in Non-linear Oscillatory Systems . . . . . . . . . . . . . 6

1.5 Vibrational Mechanics and Vibrational Rheology. Observer $\mathbf{O}$ and Observer V . . . . . . . . . . . . . . . . . . . . . . . 8

1.6 Vibrational Mechanics as a Section of the Mechanics of Systems with Hidden Motions . . . . . . . . . . . . . 13

1.7 Mistakes and Paradoxes, connected with the Interpretation of the Phenomena under Consideration. The Observer W . . . . . . . . . . . . . . . . . . . . 14

2 On the Mechanics of Systems with Hidden Motions 21

2.1 General Statements and Main Equations; Theorem 1 . . . 21

2.2 Special Cases, Examples . . . . . . . . . . . . . . . . 26

2.2.1 Mechanics of the Relative Motion . . . . . . . . . 26

2.2.2 A Solid Body with the Internal (Hidden) Degrees of Freedom . . . . . . . . . . . . . . . . . . . 27

2.2.3 Systems with Cyclic Coordinates . . . . . . . . . 31

2.2.4 Motion of a Body in a Viscous Incompressible Fluid . . . . . . . . . . . . . . . . . 34

2.2.5 Vibrational Mechanics - Systems with Hidden Fast Motions . . . . . . . . . . . . . . 36

3 Basic Statements and Mathematical Apparatus of Vibrational Mechanics

3.1 Vibrational Mechanics as the Mechanics of Systems with Hidden Motions 
3.2 Method of a Direct Separation of Motions as an

Effective General Method of Solving Problems of

Vibrational Mechanics . . . . . . . . . . . . . . . . . 38

3.2.1 Preliminary Remarks . . . . . . . . . . . . . . 38

3.2.2 The Initial Equation and Its Reduction to a System of Integro-differential Equations for the Explicit and Hidden Components of Motions . . . . . . . . . . 38

3.2.3 The Case when a Separate Equation for the Explicit Component is Obtained: Theorem 2 . . . . . . . .

3.2.4 The Main Assumption of Vibrational Mechanics, Its Formalization and Conditions of its Fulfillment; Theorem 3 and 4 . . . . . . . . . . . . . . . .

3.2.5 The Main Equation of Vibrational Mechanics. Vibrational Forces, Observers $\mathrm{O}$ and V . . . . . . 46

3.2.6 Method of an Approximate Derivation of the Expression of Vibrational Forces and of Composing the Main Equation of Vibrational Mechanics, its Grounding; Theorem 5 . . . . . . .

3.2.7 An Important Special Case; Theorem 6 . . . . . . . 50

3.2.8 On the Simplifications of Solving Equations for the Fast Component of Motion. Purely Inertial Approximation . . . . . . . . . . . . . . . . 53

3.2.9 Additional Remarks, Certain Generalizations . . . . $\quad 55$

3.2.10 Summary of the Main Relations and Results . . . . $\quad 57$

3.2.11 On the Procedure of the Practical Use of the Method . . . . . . . . . . . . . . . . 61

3.2.12 Short Bibliographic Review . . . . . . . . . . . 62

3.3 On the Main Peculiarities and Advantages of the Approaches of Vibrational Mechanics and of the Method of Direct

Separation of Motions as Compared to Other Similar Methods . . . . . . . . . . . . . . . . 63

3.3.1 Peculiarities and Limitations . . . . . . . . . . . 63

3.3.2 Advantages . . . . . . . . . . . . . . . . . . . 63

3.3.3 Final Remarks . . . . . . . . . . . . . . . . . . 65

3.4 On Other Methods of Obtaining Expressions for the

Vibrational Forces and the Main Equations of Vibrational Mechanics 
4 Potential on the Average Dynamic Systems and Extremal Signs of Stability of Certain Motions

4.1 On Potentiality on the Average and on the Extremal Signs of Stability . . . . . . . . . . . . . . . . . . . 69

4.2 Systems with Synchronized Objects. Integral Signs of Stability (Extremal Properties) of Synchronous Motions

4.2.1 Systems with Almost Uniform Rotations, Synchronization of Vibroexciters; Theorems 7 and 8 . . . . . . . . . . . . . . .

4.2.2 Systems of Quasiconservative Objects, Canonical Systems; Theorem 9 . . . . . . . . . . . . . . . . 85

4.2.3 Systems with Quasicyclic Coordinates; Theorem 10 .

4.3 Systems with Kinematic Excitation of Vibration (the Minimax Sign of Stability); Theorem 11

4.4 Systems with Dynamic Excitation of Vibration;

Theorem 12

II Vibrational Mechanics of Machines, Mechanisms and Pendulum Devices

5 Devices of Pendulum Type 101

5.1 Pendulum with a Vibrating Axis of Suspension . . . . . . 101

5.1.1 Brief Bibliographic Review . . . . . . . . . . . . 101

5.1.2 Equation of Motion . . . . . . . . . . . . . . . 102

5.1.3 Solving the Problem by the Method of Direct

Separation of Motions . . . . . . . . . . . . . . 103

5.1.4 The Use of the Mini-Max Criterion of Stability . . . 109

5.1.5 Behavior of the Pendulum Depending on the Type of Vibration . . . . . . . . . . . . . . . . 110

5.1.6 Multilink Pendulums . . . . . . . . . . . . . . 115

5.2 Chelomei's Pendulum with a Vibrating Axis of Suspension . . . . . . . . . . . . . . . . . . . . . 116

5.2.1 Brief Bibliographical Review . . . . . . . . . . . 116

5.2.2 Pendulum with an Absolutely Rigid Rod . . . . . . 117

5.2.3 The Behavior of the Washer on a Vibrating

Deformable Rod

5.3 The Follower-loaded Double Pendulum with a Vibrating Axis of Suspension 
5.4 Some Generalizations and Applications. On Vibrational Stabilization and Destabilization, Vibrational Shift (Drift) . . . . . . . . . . . . . . . . . . 130

6 Rotor Mechanisms. Machine Aggregates 137

6.1 Unbalanced Rotor on a Vibrating Base - Vibrational Maintenance of Rotation . . . . . . . . . . . . . . . 137

6.1.1 Brief Bibliography . . . . . . . . . . . . . . . 137

6.1.2 Equation of Motion, the Main Equation of Vibrational Mechanics .

6.1.3 Stationary Regimes of Rotation of the Rotor and Their Stability . . . . . . . . . . . . . . 143

6.1.4 Vibrational Maintenance of Rotation of the Rotor . . 144

6.1.5 Vibrational Capture of the Rotation of an Unbalanced Rotor of an Electric Engine. How the Efficiency of an Engine Can Be More than Unit

6.2 Devices "A Roller (Ball) in a Vibrating Cavity" and "A Ring on a Vibrating Rod (Hula-Hoop)".

Vibrational Maintenance of the Planetary Motion . . . . . 152

6.2.1 About the Devices under Consideration: a Brief Bibliographic Reference . . . . . . . . . . . . 152

6.2.2 Equation of Motion, the Main Equation of Vibrational Mechanics . . . . . . . . . . . . . . 154

6.2.3 The Discussion of Results, their Applications. The Critical Slot of Vibro-Inertial Crushing and Grinding Machines

6.3 Unbalanced Rotor (Mechanical Unbalanced Vibro-Exciter) in the Oscillatory System - Vibrational Retardation of Rotation, Zommerfield's Effect . . . . . . . . . . . .

6.3.1 On the Effects under Consideration: Brief Bibliographic Reference

6.3.2 Simplest Case: the Oscillatory Part of the System is Linear and has One Degree of Freedom

6.3.3 General Case: the Oscillatory Part of the System is Non-linear and has Several Degrees of Freedom . . . 165

6.3.4 On Certain Applications . . . . . . . . . . . . . 167

6.4 Machine Aggregates . . . . . . . . . . . . . . . . . . 168 
7 Self-synchronization of Mechanical Vibro-exciters

7.1 On the Phenomenon of Synchronization of Unbalanced Rotors (Mechanical Vibro-exciters). A Brief Review of Investigations

7.2 The Simplest Case: Self-synchronization of Vibro-exciters in the Linear Oscillatory System with

One Degree of Freedom

7.2.1 Equations of Motion and Setting up the Problem . . 180

7.2.2 Main Equations of Vibrational Mechanics . . . . . 184

7.2.3 Analysis of the Main Equations. Vibrational Torques, Partial Angular Velocities; Vibrational coupling between the rotors

7.2.4 Stationary Regimes of Synchronous Rotation and Their Stability. The Integral Sign of Stability (Extreme Property) of Synchronous Motions

7.2.5 A Case of Two Exciters. Once Again about the Phenomenon of Vibrational Maintenance of Rotation

7.2.6 About the Case of Almost Identical Exciters

7.3 The General Case. A Short Review of the

Results of Investigations

7.3.1 Equations of Motion and the Main Equations of Vibrational Mechanics in the General Case . . . . .

7.3.2 Potential Function and Integral Criterion of Stability

7.3.3 Short Review of the Results on the Theory of Synchronization of Mechanical Vibro-exciters

7.4 Steadiness of Phasing the Vibro-exciters and the Adaptive

Property of Vibrational Machines in case of Self-synchronization

7.4.1 On the Concept of the Steadiness of Phasing . . . . 202

7.4.2 Estimation of Steadiness. On the Relative Strength of Vibrational Coupling between the Unbalanced Rotors

7.4.3 The Adaptive Property of Vibrational Machines with Self-synchronized Vibro-exciters 
7.5 On Theoretic Investigation of the Devices with Self-synchronized Vibro-exciters. An Example of Using the Integral Criterion of Stability . . . . . . . . . 206

7.6 On the Synthesis of the Devices with Self-synchronized Vibro-exciters . . . . . . . . . . . . . . . . . . 209

7.7 Regularities and Paradoxes of Self-synchronization of Unbalanced Vibro-exciters . . . . . . . . . . . . . . . 210

7.8 Supplement: On the Phenomena of Synchronization of the Oscillatory and Rotational Motions in Nature and in Technology. The modern state of the problem. General Definition of synchronization . . . . . . . . . 214

8 Generalized Principle of Auto-balancing

8.1 Laval's Principle and its Generalization, Ensuing from the Theory of Self-synchronization of Mechanical Vibro-exciters and from Some Other Investigations

8.2 Application to the Theory of Group Foundations for the Unbalanced Machines . . . . . . . . . . . . . . 224

8.3 Supplement to the Theory of Auto-balance Devices . . . . 227

\section{Vibrational Mechanics of Processes}

(Vibrational Displacement and Shift)

9 The Main Models and General Regularities of Processes of Vibrational Displacement from the Position of Vibrational Mechanics

9.1 On the Effect of Vibrational Displacement, its Theory and Applications . . . . . . . . . . . . . . . . . . .

9.2 Simplest Model of the Process of Vibrational Displacement

9.2.1 Motion of a Particle over a Rough Horizontal Plane under the Action of a Longitudinal Harmonic Force or the Longitudinal Vibration of the Plane . . . .

9.2.2 Analysis of the Solutions. Effect of the Seeming Vibrational Transformation of Dry Friction into Viscous. The Driving and the Vibro-transformed Vibrational Forces

9.3 Physical Mechanisms and Main Types of Asymmetry of the System, Causing Vibrational Displacement 
9.4 More Complicated Main Models and Problems of the

Theory of Vibrational Displacement

9.4.1 One-dimensional Motion of the Particle in the

Resistant Medium under the Action of Vibration

$[95,99,198,564$, vol. 2 and 4$]$. . . . . . . .

9.4.2 The Problem in 9.4.1 in the Case of

Harmonic Vibration

9.4.3 The Motion of a Particle over the Tilted Rough

Plane, Performing Harmonic Oscillations in Two Mutually Perpendicular Directions . . . . . . .

9.4.4 On Vibrational Transportation of a Body (Particle)

Upwards along the Tilted Plane. The Limiting

Angle of Rise.

9.4.5 About Other Models and Problems . . . . . . . . 260

10 Effects of Vibrational Displacement in Technique, Technology and in Nature

10.1 Vibrational Transportation

10.2 Vibrational Separation of the Components of

Granular Mixtures

10.2.1 Factors, Determining the Efficiency of Using

Vibration in the Processes of Separating the

Components of Granular Mixtures

10.2.2 On Separation of Particles in a Layer of Granular

Material under the Action of Vibration (Segregation,

Stratification, Self-classification) . . . . . . .

10.2.3 General Statement of the Problem on the Separation

of Particles of the Granular Mixture in a

Vibrating Vessel. Brief Characteristic of the

State of the Problem

10.2.4 Motion of a Heavy Particle in the Medium with a

Resistance of Dry Friction Type, Performing

Circular Horizontal Oscillations.

Pseudo-resonance Effect

10.2.5 Problem of 10.2.4 in the Case of Vertical Harmonic

Oscillations of the Medium. The Effect of Floating up of a Heavy Particle in the Medium of Light

Small Particles 
10.2.6 Kinetics of Vibrational Separation of Multi-Component Mixture (the Continual Description) . . . . . . . 280

10.2.7 Separation of Particles on the Vibrating Surfaces . . 287

10.2.8 On Separating the Particles in the Vibrational and

Wave Fields, Created in the Rarefied Suspensions . . 291

10.3 Vibrational Sinking and Intrusion, Vibrational Cutting . . 292

10.4 Vibrational Transformation of Motion; Vibro-engines . . . 298

10.5 Vibrational Movement, Vibrational Coaches . . . . . . 303

10.5.1 Definitions. Preliminary Remarks . . . . . . . . . 303

10.5.2 Vibrational Movement over a Rough Surface.

Self-propelled Vibro-compactors of the Ground,

Travel on Skateboards . . . . . . . . . . . . . 304

10.5.3 Vibrational Movement in Fluid and in Gas.

Vibro-flier, the movement of living organisms . . . . 306

10.5.4 Vibrational Movement in Heterogeneous Force

Fields. Gravi-flier, Magneto-flier . . . . . . . . . 311

10.6 The Vibro-jet Effect, Vibrational Pumps; Some More about Vibrational Coaches . . . . . . . . . . . . . . 313

10.6.1 On Vibro-jet Effect, Vibrational Pumps . . . . . . 313

10.6.2 Lishansky's Vibrational Pump; The Simplest

vibroflighter: An Approximate Theory . . . . . . . 317

10.6.3 Generating the Flow of Fluid by Means of a

Vibrating Plate with Holes of

Changing Cross-sections . . . . . . . . . . . . . 319

10.6.4 Five Principle of Generating Vibrational Flows

and Forces . . . . . . . . . . . . . . . . . 322

10.6.5 On Vibrational Convection . . . . . . . . . . 324

10.6.6 On the Main Equations of Vibrational

Hydromechanics and Hydraulic . . . . . . . . . . 324

11 Vibrational Shift (Drift) 325

11.1 On the Notion of Vibrational Shift (Drift) . . . . . . . . 325

11.2 Effect of Vibrational Shift in Applications; Special Features of the Effect in Systems with Dry Friction . . . . . . . . . 325

IV Vibrorheology

12 On Rheology and Vibrorheology $\quad 331$

12.1 Rheology as a Section of Mechanics . . . . . . . . . . . 331 
12.2 Definition of Vibrorheology. Macro- and Microvibrorheology . . . . . . . . . . . . . . 332

12.3 Vibrorheological Equations, Vibrorheological Properties and Effective Vibrorheological Characteristics . . . . . . .

13 Effective Rheological Characteristics under the Action of Vibration

13.1 Effective Coefficients of Dry Friction under the Action of Vibration or Shock; Some Applications

13.1.1 Effective Frictional Coefficients of Rest. The simplest Model - an Absolutely Solid Body under a Harmonic Action

13.1.2 A More Complicated Model is a Solid Body with an Internal Degree of Freedom . . . . . . . . . . . . 337

13.1.3 Several Other Models; Shock Effect; Experiment of Tolstoy

13.1.4 Vibrational Conception of the Sliding Friction; Vibrational Control of Dry Friction

13.1.5 Application to the Theory and to Projecting Designs, Operating in Conditions of Impacts and Vibration

13.1.6 Possible Applications to Seismology and to the Theory of Explosive Effects

13.2 Effective Friction when the System with a Positional-Viscous Resistance is acted upon by Vibration

13.2.1 Vibrational Transformation of the Characteristic of the Resistance of the Oscillatory System with One Degree of Freedom

13.2.2 Special Cases - the Asynchronous Suppression and Excitation of Self-oscillations; Some Applications . .

13.3 Equation of Reinolds' as a Vibrorheological Equation.

Effective Viscosity of Fluid in Turbulent Motion; Effect of the External Vibrational Action

13.4 On Other Cases of Using Concepts of Effective Viscosity under Vibration

14 Vibrorheological Transformation of Nonlinear Mechanical Systems with Discontinuous Characteristics into Systems with Viscous Friction 
14.2 Vibrorheology of Systems with Periodic Collisions

14.2.1 On Vibrorheological Simulation of Vibroshock

Interactions by Forces of Viscous Friction

14.2.2 Applications to the Calculation of the Capacity of Crushers .

15 Vibrorheology of Granular Materials

15.1 Vibrorheological Models of a Layer of Granular Medium . . 363

15.2 Some Applications . . . . . . . . . . . . . . . . . . 372

15.2.1 The Process of Vibrobunkering of Granular

Materials

372

15.2.2 Motion of a Layer of Granular Material in a Rectangular Tray Whose Bottom is Vibrating

Heterogeneously in the Transverse Direction (to the

Theory of Vibrational Screens with an

Elastic Resonant Sieve)

376

15.2.3 Motion of the Loading in Vibrational Mills and

Instruments for the Volume Vibrational Treatment

of Machinery Parts . . . . . . . . . . . . . . . 382

15.2.4 On Supplements to the Theory of Crushers . . . . . 382

15.3 On the Behavior of the Granular Material in the

Communicating Vibrating Vessels . . . . . . . . . . . . 383

16 Penetration of Vibration into Certain Media 387

16.1 Preliminary Remarks . . . . . . . . . . . . . . . . . 387

16.2 Penetration of Vibration into a Viscous Fluid . . . . . . . 387

16.3 On the Penetration of Vibration into Suspensions . . . . . 393

16.4 Penetration of Vibration into Granular Material . . . . . 394

16.4.1 The Case of Circular Oscillations of a Horizontal

Plate in its Plane . . . . . . . . . . . . . . . . 394

16.4.2 The Case of Rectilinear Longitudinal Oscillations of a Horizontal Plate . . . . . . . . . . . . . . . 397

16.4.3 The Case of Transverse Oscillations of the Plate . . 397

16.5 On Penetration of Vibration into Concrete Mixes . . . . . 399

16.6 On the Theory of Vibro-conduction . . . . . . . . . . . 399

17 Microvibrorheology: the Behavior of Suspension under Vibration, Effective Viscosity and Effective Density of Suspension

17.1 Preliminary Remarks 
17.2 Structureless Suspensions - Solid Particles in a Viscous Fluid . . . . . . . . . . . . . . . . . . . 403

17.3 Structurized Suspensions - Particles in the Medium with the Resistance of the Type of Dry Friction . . . . . . . . 407

18 The Problem of the Control of Vibrorheological Properties of Mechanical Systems. The Idea of Creating Dynamic Materials

18.1 On the Problem of Forming the Properties of Nonlinear Mechanical Systems by Means of Vibration . . . . . . . . 411

18.2 On Dynamics Materials . . . . . . . . . . . . . . . . 412

18.2.1 The Idea and Definition of Dynamic Materials . . . 412

18.2.2 Two kinds of Dynamic Materials . . . . . . . . . 413

18.2.3 About Some Possibilities of the Implementation of Dynamic Materials . . . . . . . . . . . . . 413

18.2.4 On Some Potentialities Provided by Using the Dynamic Materials . . . . . . . . . . . . . . 417

18.2.5 On Dynamic Surfaces . . . . . . . . . . . 418

19 Supplements

19.1 Vibrorheological Effects in Macroscopic Homogeneous Media (Turbulent Viscosity, Vibrocreep, Vibrorelaxation, Vibroplasticity, Fatigue of Materials)

19.2 Certain General Vibrorheological Regularities . . . . . . .

V Some Other Problems

20 The Motion of the Particle in a Fast Oscillating Nonuniform Field

20.1 The simplest Case: Rectilinear Motion in a Field of a

Harmonic Standing Wave

20.2 Special Case: a Pendulum with a Vertically Vibrating Axis of Suspension

20.3 Generalization of the Problem

21 Resonance (Synchronization) in Orbital Motions of Celestial Bodies

21.1 Preliminary Remarks

21.2 Main Results of the General Theory of Synchronization and of the Theory of Potential on the Average Dynamic Systems as Applied to the Problem under Consideration . 
21.2.1 Description of the System. The Initial System and the Frame System . . . . . . . . . . . . . . .

21.2.2 The Potential Function and the Integral Sign of Stability (Extreme Property) of Resonant Motions of the System of Bodies . . . . . . . . . . . . . . . 439

21.2.3 On the Theoretic Explanation of the Natural Prevalence of Resonance in the Solar System . . . . 441

21.2.4 On the "Principle of the Least Interaction" by Ovenden, Feagin and Graff and on the Role of Dissipative Forces in the System . . . . . . . .

21.3 The Case when the Orbits of Bodies Lie in the Same Plane or in Proximate Planes and Have Small Eccentricities. On classification of resonances . . . . . . . . . . . . . .

21.4 The Case of Two Revolving Bodies: Comparison with the Results of the Direct Analytical Investigation and with the Observed Data . . . . . . . . . . . . . . . . .

21.5 On Resonances in the Solar System. Hypothesis about Simple Resonancity . . . . . . . . . . . . . . . . . . 450

21.6 On Other Problems (Motion of Celestial Bodies with the Internal Degrees of Freedom) . . . . . . . . . . . . . . 452 Bibliography Index 